\title{
ELECTRON BEAM DUMP PARTICLE SEARCH
}

\begin{abstract}
A proposal to search for short-lived particles that couple to the electron by observing their decay in flight downstream from an electron beam dump.
\end{abstract}

M. CRISLER, H. FENKER, I. LEEDOM, S. PORDES

Fermi National Accelerator Laboratory

May 30, 1986

Scientific Spokesperson: M. Crisler, Fermilab-MS122, Tel.(312)840-4099 


\section{ABSTRACT}

The debate over the existence of a new particle postulated to explain the narrow positron spectra seen in heavy ion collisions has focused attention on a region of mass/lifetime where such a particle may exist and yet would not have been seen.

To obtain the best possible sensitivity to elementary particles coupling to the electron in this unexplored region, we propose an electron beam dump experiment which will make parasitic use of the newly constructed wide band electron beam. 


\section{INTRODUCTION}

The anomalous production of $340 \mathrm{KeV}$ positrons in heavy ion collisions is by now a well established phenomenon ${ }^{1}$, lacking a conventional nuclear physics interpretation. The explanation of this effect as the production and decay of a $1.8 \mathrm{MeV}$ neutral particle ${ }^{2}$ is supported by the recent observation at the GSI in Darmstadt of coincident electron-positron pairs having equal lab energies $\left(\mathrm{E}_{\mathrm{e}^{+}}^{+}\right.$ $\left.\mathrm{E}_{\mathrm{e}^{-}}=1.8 \mathrm{MeV}\right)$. Attempts to identify this particle as the axion have led to a model by Krauss and Wilczek ${ }^{4}$ which predicts an elementary pseudo-scalar that couples preferentially to the electron.

The phenomenology of such particles has been examined and two complementary constraints have been determined on their lifetime. First, an elementary pseudo-scalar coupling to the electron contributes to its anomalous magnetic moment. This leads to an upper bound on the coupling strength which in turn determines a lower bound on the lifetime $\left(3 \times 10^{-14} \mathrm{sec}\right.$ for $1.8 \mathrm{MeV}$ mass). An upper bound on the lifetime comes from proton beam dump experiments where the particle would be radiated from electrons produced in the cascade process $\mathrm{pN} \rightarrow \pi^{\circ} \rightarrow \gamma \rightarrow \mathrm{e}^{+} \mathrm{e}^{-}$and, for sufficiently long lifetimes, would escape from the dump before decaying. Based on the absence of large $x$ electron-positron pairs, Fermilab experiment E605 places an upper limit of $1.5 \times 10^{-14} \mathrm{sec}$ on the lifetime.

As a function of mass and lifetime, the experimentally allowed region for an elementary pseudo-scalar is shown in Figure 1 . While the combination of $(g-2) e$ and the results of proton beam dump experiments has probably ruled out the "Darmstadton" as a conventional point-like particle, interest in it has revealed a region of mass/lifetime where such particles could exist and yet would not have been found.

Experiment 774 will explore this region and will set the definitive mass and short lifetime limits on point-like particles coupling to the electron. 


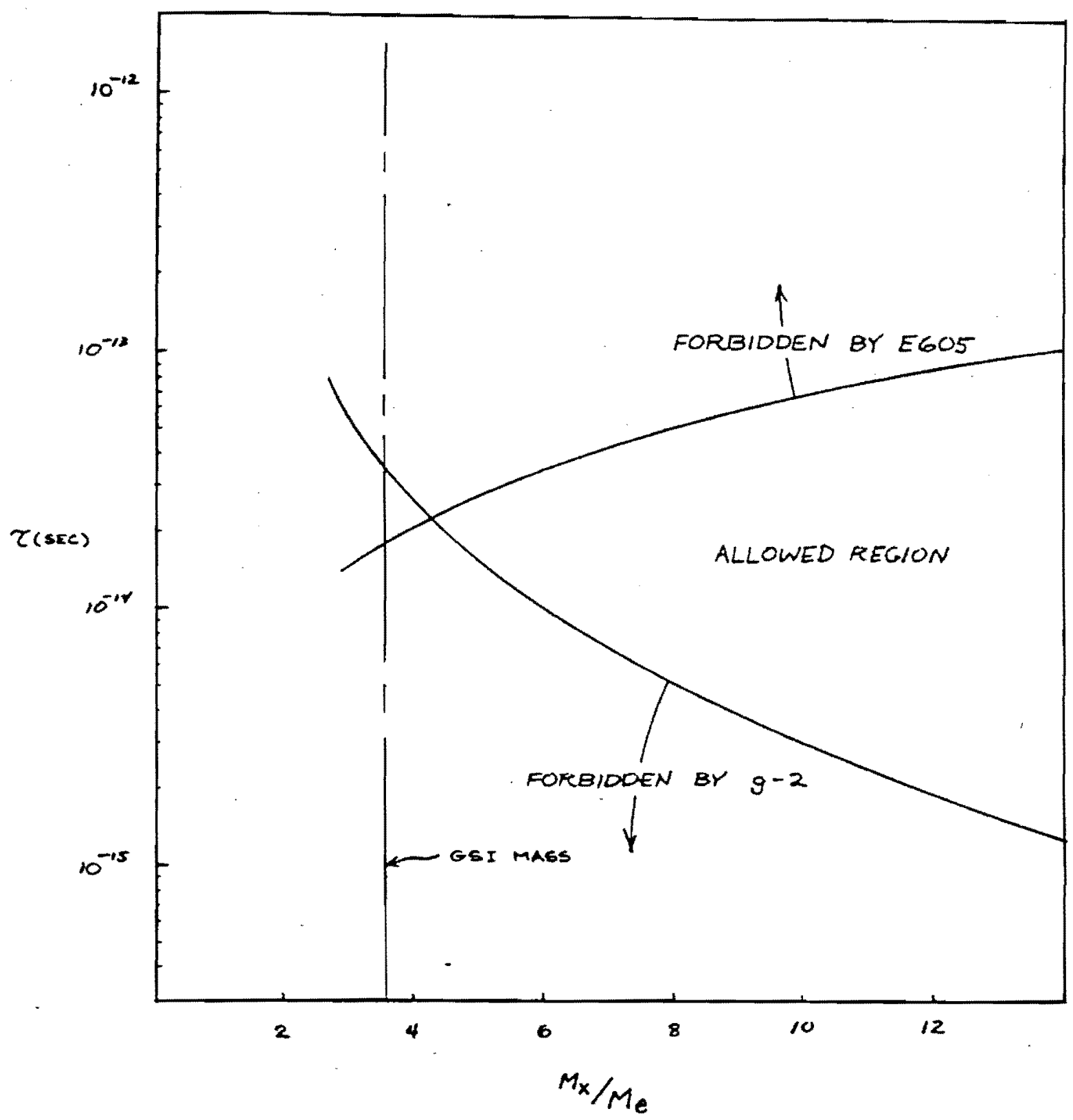

Figure 1: Allowed region of mass and lifetime for an elementary pseudo-scalar. 


\section{CHARACTERISTICS OF PSEUDO-SCALAR PRODUCTION BY ELECTRONS}

An elementary particle that couples to the electron can be produced by bremsstrahlung as shown in Figure 2. The cross-section for bremsstrablung production of a pseudo-scalar has been calculated' and is characterized by two important features. The radiated particle carries away most of the electron's momentum, and it is produced at a very small characteristic angle 1.4 $\mathrm{M}_{\mathrm{x}} / \mathrm{E}_{\text {beam. }}$. The factor of 1.4 arises because the mean energy of the radiated particle is about $70 \%$ of the primary beam energy even after folding in the degradation of the primary electron's energy in the dump.

The signature for pseudo-scalar production in beam dump is thus quite distinctive: an electron-positron pair having most of the primary beam energy materializes downstream of a "totally absorbing" dump.

Figure 3 shows the predicted momentum distribution for the electron-positron pairs from the decay of a pseudo-scalar produced by a high energy electron beam incident on a tungsten dump. The total production rate is approximately $6 \mathbf{x}$ $10^{-21} / \tau$ per incident electron."

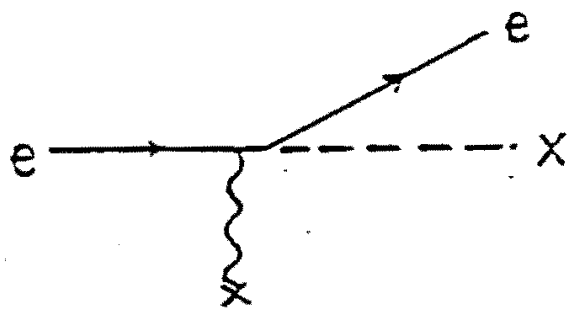

Figure 2: Bremsstrahlung production Feynman diagram. 


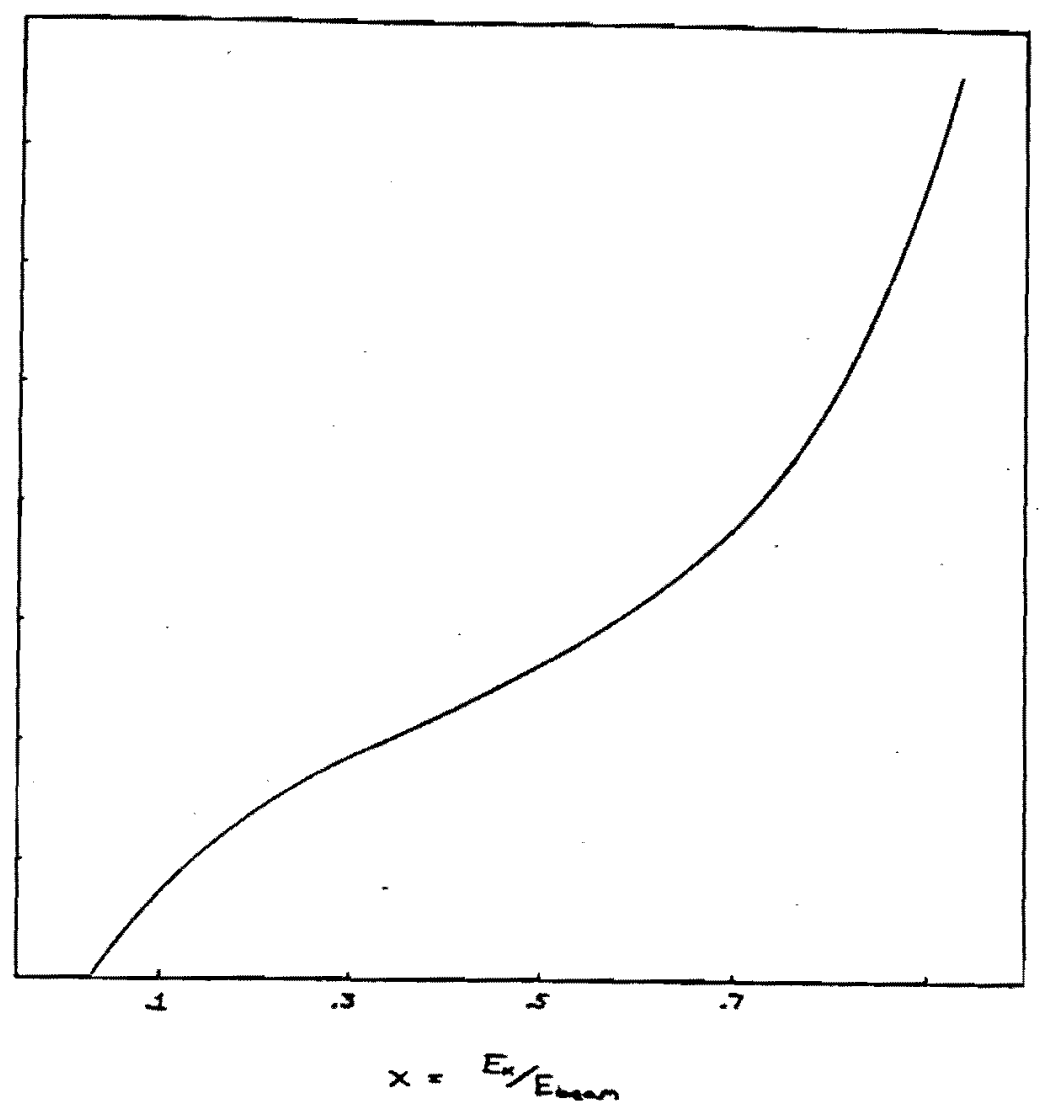

Figure 3: Momentum distribution of pseudo-scalars produced in an electron beam dump. 


\section{WHY AN ELECTRON EXPERIMENT AT FERMILAB?}

The arithmetic of beam dump searches for short-lived particles suggests that the ideal situation involves a very high energy beam to give the produced particles a large Lorentz factor and a very short dump to maximize the chance that the particles escape the dump before decaying. The region of mass-lifetime space for which a search is sensitive is determined by the energy of the beam and length of the dump and is comparatively insensitive to the accumulated luminosity.

The choice of an electron beam has three attractive features. First, since the particles in question decay into electron-positron pairs with a particular lifetime and mass, their production rate by electron bremsstrahlung can be calculated and unknown quantities such as the coupling to hadrons can be ignored when deriving limits. Second, in an electron beam dump, the pseudo-scalars produced carry most of the incident beam energy as opposed to backgrounds which are typically at small $x$; this leads to a natural separation between signal and background. Third, to absorb an electron beam it is practical to use a short dump, 10-20 cm of tungsten, compared to the 2 to 4 meters needed to absorb a hadron beam.

A compelling motivation behind this proposal is the opportunity to make parasitic use of the highest energy electron beam in existence'. This electron beam was constructed at Fermilab for use by experiment E687 as a high-energy photon source and has been operated with electron energies up to $600 \mathrm{GeV}$. In normal operation, after passing through a thin $\mathrm{Pb}$ radiator to produce photons, the electron beam is swept laterally and dumped. This situation naturally lends itself to a parasitic experiment which makes use of these high energy electrons.

If we assume that the normal operating mode of experiment E687 is $10^{7}$ electrons/sec @ $450 \mathrm{GeV}$, then the sensitivity that can be obtained by this search (with a 20cm dump) is shown in Figure 4. 


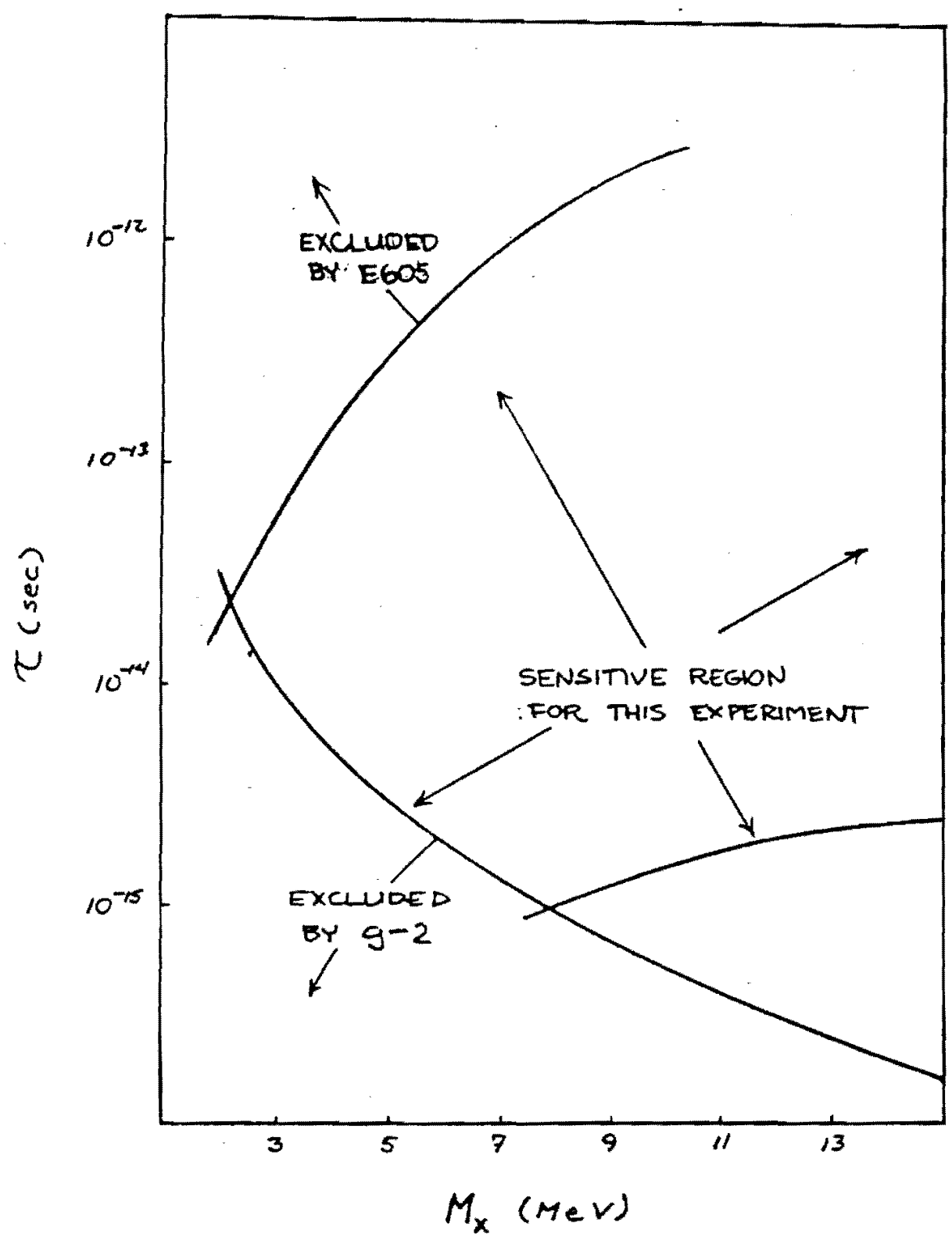

Figure 4: Sensitive region for this experiment. 


\section{PROPOSED LAYOUT AND APPARATUS}

Our experiment depends crucially on the new high-energy electron beam designed for the photo-production experiments in the Proton Area and will produce minimal interference with the already approved experiment, E687. The photons for E687 are produced by passing a high-energy electron beam through a $\sim 15 \%$ radiator; the photons produced in this radiator pass into the E687 apparatus while the parent electrons are swept into a dump by a series of magnets. We propose to replace a section of the existing dump with a variable collimator which will pass electrons within a selected momentum band to a tungsten-scintillator active beam dump (Figure 5). By selecting particles with $\sim 2 / 3$ of the primary momentum, we will reduce the hadron contamination of the primary beam to $\sim 10^{-3}$. Following the dump will be a veto counter, an evacuated decay pipe, and a trigger counter to tag the decay in flight of a neutral particle. Charged tracks from the decay will be momentum analyzed by a magnetic spectrometer and an electromagnetic calorimeter at the end will provide an energy measurement for energy/momentum (E/P) comparison. This comparison is critical for rejection of background $e^{+} e^{-}$pairs coming from the decay $\mathrm{K}_{\mathrm{s}}^{\mathrm{O}} \rightarrow \pi^{0} \pi^{\circ} \rightarrow \mathrm{ye}^{+} \mathrm{e}^{-}$. The kinematics of this process require that the trailing $\pi^{\circ}$ have at least $8 \%$ of the kaon momentum, facilitating a clean rejection by an E/P cut. The detector is shown in Figure 6; a list of its major components is given in Table 1.

We assume the following running conditions:

electron beam intensity:

hadron fraction in primary beam

P774 electron rate:

hadron fraction in P774 beam:

total flux (10 wk. parasitic running):

electron-nucleus luminosity:
$10^{7}-10^{8}$ electrons/sec

$10 \%$

$10^{6}$ electrons/sec

$10^{-3} /$ electron

$10^{12}$ electrons, $10^{9}$ hadrons

-few events per nanobarn 


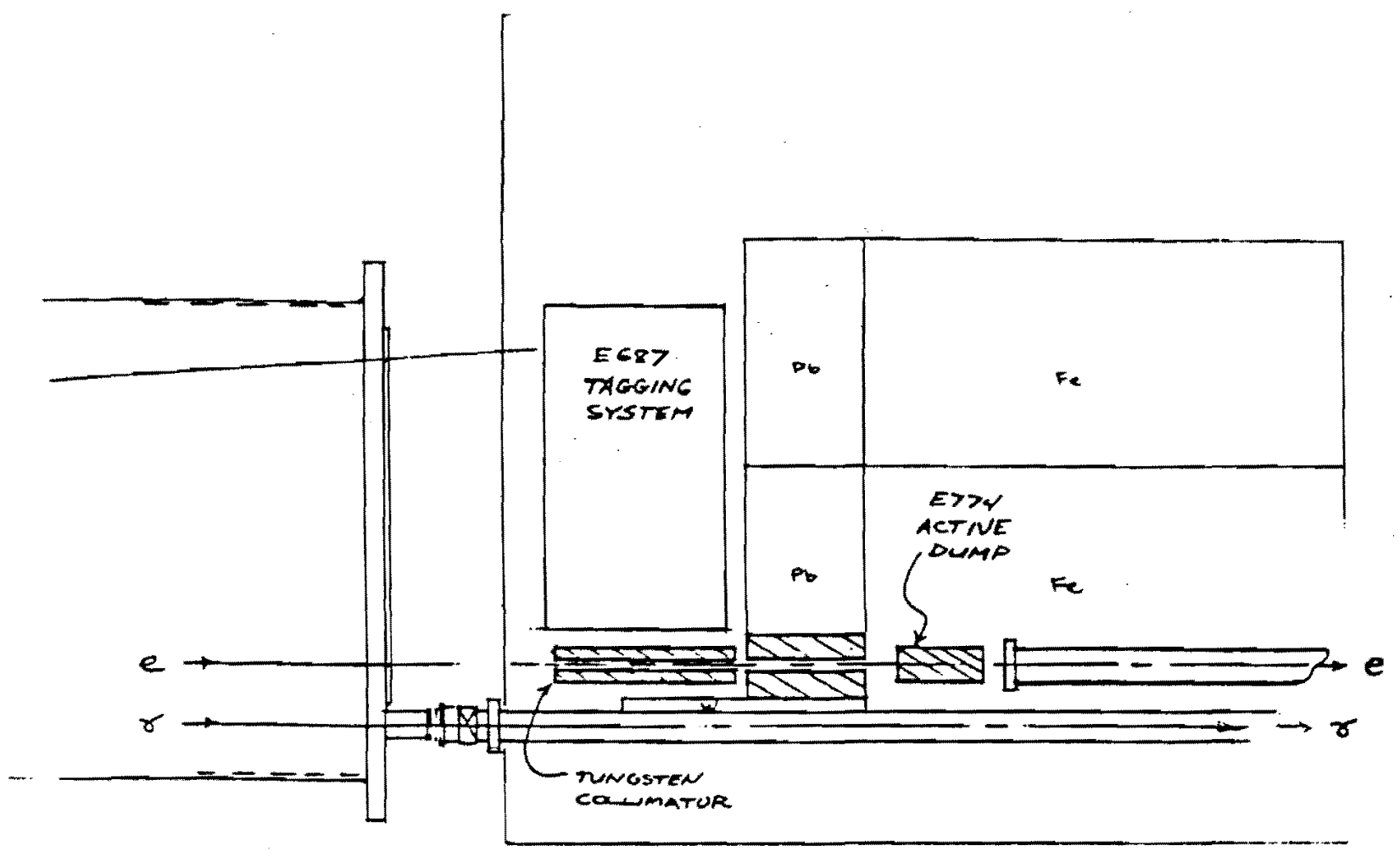

Figure 5: Modification to the E687 beam dump. 


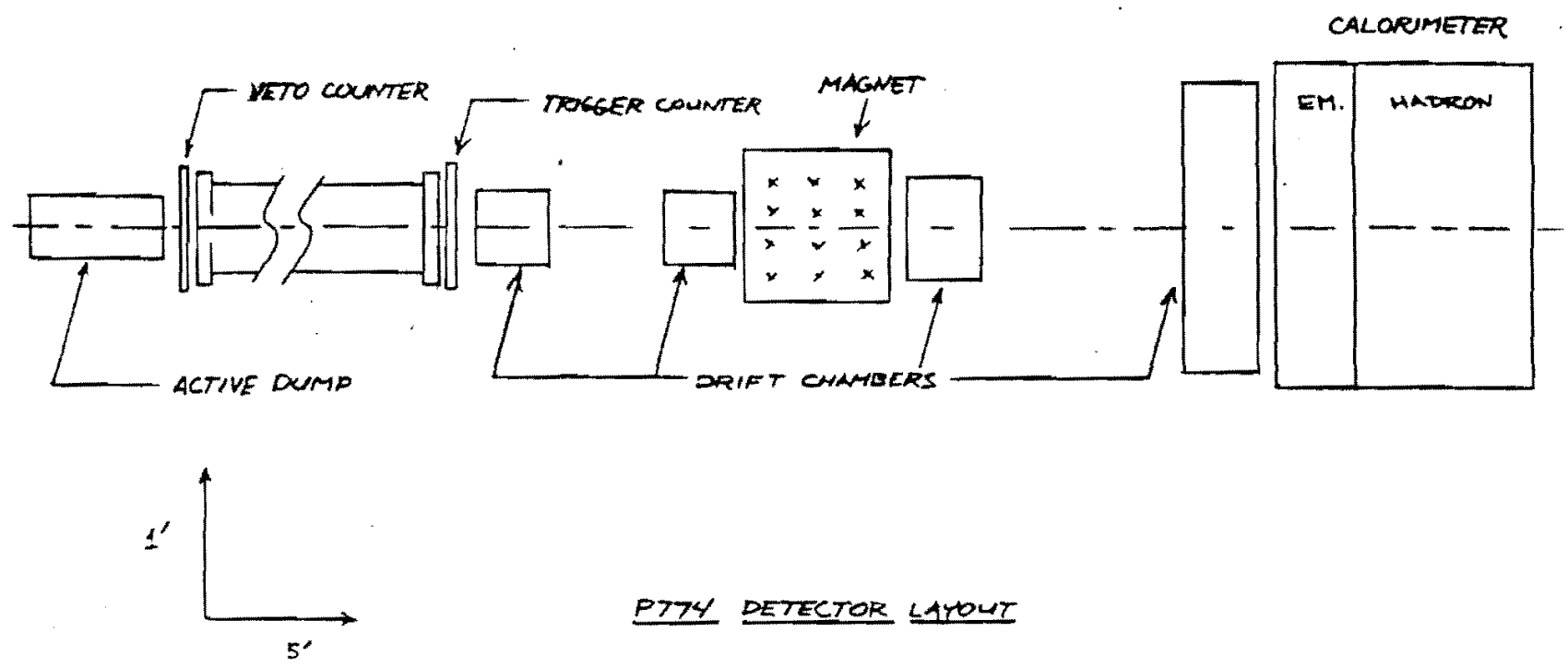

Figure 6: Proposed detector arrangement. 
Table 1: SUMMARY OF DETECTOR ELEMENTS

1) Active dump

2) Veto counter

3) Decay pipe

4) Trigger counter

5) Upstream drift chambers

6) Magnet

7) Downstream drift chambers

8) Electromagnetic calorimeter

9) Hadron calorimeter $2^{\prime \prime} \times 2^{n} \times\left(8^{n}-16^{n}\right)$ tungsten-scintillator calorimeter.

Function: electron absorber, provides measurement of spatial distribution of energy deposition.

$2 " \times 2 "$ plastic counter(s)

Function: vetos on charged particles emerging from the dump.

$3^{n}$ diameter $x$ 10' evacuated beam pipe

Function: evacuated decay space

$2^{\prime \prime} \times 2^{\prime \prime}$ scintillation counter(s)

Function: trigger signal for decay in flight.

$2^{n} \times 2^{\prime \prime}$ active area drift chambers

2 possibilities*

$4 " x 6^{\prime \prime}$ and $4^{\prime \prime} \times 20^{\prime \prime}$ active area drift chambers.

6" $\mathrm{x}$ 20" active area $\mathrm{Pb}$-scintillator or $\mathrm{Pb}$-glass calorimeter

Function: trigger signal and precision measurement of electromagnetic energy.

$12^{\prime \prime} \times 20^{\prime \prime}$ active area $\mathrm{Fe}$-scintillator calorimeter

Function: identify hadrons

The detector uses about 40 photomultiplier tubes and 250 channels of wirechamber electronics.

* since our active area is only $8^{\prime \prime}$ away from the photon beam, this is either a small, custom magnet which fits in the required space, or a larger gap magnet like a BM109 which can contain both beam pipes. 


\section{DISCUSSION OF TRIGGER AND BACKGROUNDS}

This discussion is based on an exposure of $10^{12}$ electrons $\left(10^{9}\right.$ hadrons). To set a scale, this would produce about $10^{5}$ pseudo-scalars of mass $10 \mathrm{MeV}$ and lifetime $10^{-14}$ seconds. We will use a veto counter at the end of the active dump and a trigger counter at the end of the decay-pipe to restrict our triggers to events in which no charged particle emerges from the dump and a neutral particle decays to charged particles within the decay-pipe. Since we shall also require a substantial energy deposition in the electromagnetic calorimeter, our backgrounds will result only from hadronic decays in the decay volume which produce a high energy electron-positron pair. We have considered various processes which could result in low mass electron-positron pairs at large $X$ and have identified two as dominant. Reactions which result in a high energy $\pi^{\circ}$ materializing in the decay volume will be a source of electron-positron pairs from Dalitz decay. Second, reactions in which a high energy photon emerges from the dump will produce low mass electron-positron pairs if the photon converts in the trigger counter.

SOURCES OF LARGE $X \pi^{\circ}, \mathrm{s}$

Since large $\mathrm{X} \pi^{\mathrm{O}}$, s are kinematically forbidden in hyperon decays, the only source of concern is the decay $K \underset{\mathrm{s}}{\rightarrow} \pi^{0} \pi^{0}$. We have considered kaons photo-produced by bremsstrahlung photons in the dump and from the interactions of beam pions. The photon luminosity per incident electron in a tungsten dump, $\mathrm{dL} / \mathrm{dX}$, is $-2.8 \times 10^{-8} \times\left(\log _{e}(\mathrm{X}) / \mathrm{X}\right)$ per microbarn where $\mathrm{X}$ is $E_{\gamma} / E_{e}$ (see Appendix 1). Multiplying this by the photoproduction cross-section per tungsten nucleus $\left(120 \times \mathrm{A}^{0.9}\right.$ microbarns) gives a photoproduction rate, $\mathrm{dN} / \mathrm{dX}$ of $-3.7 \times$ $10^{-4}\left(\log _{\mathrm{e}}(\mathrm{X}) / \mathrm{X}\right)$. Above $\mathrm{X}$ of 0.5 , this implies $8.9 \times 10^{-5}$ interactions per incident electron. Since this rate is small compared to the interaction rate of beam hadrons (which are by definition at $\mathrm{X}=1$ ) inclusive $\mathrm{K}^{\circ}$ photo-production 
will be ignored. Diffractively produced phi mesons are a copious source of $\mathrm{K}_{\mathrm{s}}^{\circ}$ but these kaons all have $\mathrm{X}$ below 0.6 .

The cross-section for $\pi^{-} \mathrm{Be} \rightarrow \mathrm{K}_{\mathrm{s}}^{0}+\mathrm{X}$ has been measured ${ }^{10}$ and can be parameterized as $\mathrm{d} \sigma / \mathrm{d} x=3 \mathrm{mb}(1-\mathrm{x})$ for $x>0.5$. Assuming an $\mathrm{A}^{2 / 3}$ dependence, the $\mathrm{K}_{\mathrm{B}}^{\mathrm{O}}$ production rate above $\mathrm{X}=0.5$ is $2.5 \times 10^{-3}$ per berm $\pi$ giving a total of 2.5 $x 10^{6} \mathrm{~K}_{\mathrm{s}}^{\mathrm{o}}$ per $10^{12}$ electrons. This has to be multiplied by the appropriate decay and kinematic probabiities as shown below and results in no events that survive the analysis.

$$
\begin{aligned}
& \text { Rate for: } \pi^{-}+\mathrm{W} \rightarrow \mathrm{K}^{\mathrm{O}}+\mathrm{X} \\
& \mathrm{K}_{\mathrm{s}}^{\mathrm{O}} \text { production rate }(\mathrm{x}>0.5)=2.5 \times 10^{-3} / \pi^{-} \\
& \text {total number of } \mathrm{K}_{\mathrm{s}}^{\mathrm{O}}=2.5 \times 10^{6} / 10^{12} \text { electrons } \\
& \text { number escaping dump }=6.5 \times 10^{5} / \\
& \text { number decaying in decay volume }=1.2 \times 10^{5} / \text { " } \\
& \text { number decaying to } \pi^{\circ} \pi^{\circ}=3.7 \times 10^{4} / \\
& \text { number of Dalitz decays } \pi^{\circ} \mathrm{\gamma e}^{+} \mathrm{e}^{-}=8.9 \times 10^{2} / \text { " } \\
& \text { number with } \mathrm{E}_{\mathrm{e}^{+}} \mathrm{e}_{-} 0.9 \mathrm{E}_{\mathrm{Ko}} \quad=3.6 \times 10^{+1} / \text { " } \\
& \text { number surviving } E / P \text { cut } \quad .36 \text { events } / 10^{12} \text { electrous }
\end{aligned}
$$

\section{SOURCES OF LARGE X PHOTONS}

Processes which result in a large $X$ photon emerging from the dump are hard bremsstrahlung from a beam pion or pion charge-exchange $\pi^{-} \mathrm{P} \rightarrow x^{\circ} \mathrm{N}$ followed by the decay $x^{\circ} \rightarrow \gamma \gamma$. In either case, the reaction must occur near the end of the dump for the photon to escape. We calculate that the number of events from bremsstrahlung in which one photon has $X>0.5$, survives the dump, and converts in the trigger counter is 6 . For such events to survive the analysis, the original pion must also be undetected by the veto counter. Consequently, there is no background from this source. 
Pion charge-exchange will actually produce a dozen event candidates which survive the E/P cut. They will, however, have the well-defined signature of a single minimum ionizing particle in the active dump and should be easily distinguished from real events in which a reasonable fraction of the beam energy is deposited in the first few $\mathrm{cm}$ of the dump.

\section{SUMMARY}

We have designed an apparatus with sufficient power to recognize and reject the important backgrounds to a definitive search for low mass particles that couple to the electron. The experiment makes parasitic use of a newly constructed beam line. While the detector is, conceptually, a conventional spectrometer (active target, magnetic spectrometer, calorimeters), the kinematics of the signal allow each element to be constructed with a small active area and the apparatus to be equipped with a manageably small number of channels. 


\section{REFERENCES}

1. J. Schweppe et al, Phys. Rev. Lett. 51, 2261(1983); M. Clemente et al., Phys. Lett. 137B, 41 (1984); T. Cowan et al., Phys. Rev. Lett. 54, 1761 (1985).

2. A. Balantekin et al., Phys. Rev. Lett. $\underline{55}, 461$ (1985).

3. T. Cowan et al., Phys. Rev. Lett. 트, 444 (1986).

4. L. M. Krauss and F. Wilczek, Yale preprint YTP-86-03, submitted to Phys. Rev. Lett., February, 1986.

5. J. Reinhardt et al., Phys. Rev. C33, 194 (1986).

6. C. Brown, Fermilab Beam Dump Workshop, 20 March, 1986.

7. Y. S. Tsai, private communication; B. Lu, private communication.

8. R. Arnold et al,, SLAC Proposal E-141, March, 1986.

9. J. Butler et al., Fermilab Technical Memo TM-963, April, 1980.

10. R. T. Edwards et al., Phys. Rev. D18, 76 (1978). 


\section{APPENDIX 1: LUMNOSITY IN AN ELECTRON BEAM DUMP:}

The physics of an electron beam dump experiment is determined by the spectrum of electron and photon momenta produced in the electromagnetic shower.

The energy of the primary electron is continuously degraded, so its luminosity spectrum is expressed in terms of the electron path length distribution (path length per unit momentum fraction), $d Z / d X$. The luminosity is given by

$$
d L_{e} / d X=\rho N / A(d Z / d X)
$$

where $\rho$ is the density, $N$ is Avogadro's number, and $A$ is the atomic weight. The photon, on the other hand has a well-defined energy and mean-free-path, so its luminosity is given by

$$
d I_{\gamma} / d X=\rho N / A \quad \delta Z\left(d N_{\gamma} / d X\right)
$$

where $\mathrm{dN}_{\gamma} / \mathrm{dX}$ is the photon spectrum. At large $\mathrm{x}$, the electron path length distribution is dominated by the path of the primary electron and the photon spectrum comes from the radiation of the primary electron. Using the approximation that the bremsstrahlung spectrum is $1 / \mathrm{X}$, the photon spectrum produced in the dump is given by

$$
\mathrm{dN}_{\gamma} / \mathrm{dx}=-\ln \mathrm{x} / \mathrm{X}
$$

The electron path length distribution has been computed numerically, and for large $X$ can be parameterized as

$$
\begin{aligned}
\mathrm{dZ} / \mathrm{dX} & =\quad 1.1 \lambda(0.5<\mathrm{X}<0.95) \\
& =\quad 5.6 \lambda(\mathrm{X}>0.95)
\end{aligned}
$$

where $\lambda$ is the radiation length of the absorber. (To understand these expressions, they imply, for example, that the primary electron travels, on average, $0.28 \lambda$ before losing more than $5 \%$ of its energy $(5.6 \lambda \times 0.05)$.) 
This leads to simple formulae for the electron and photon luminosity in a tungsten beam dump.

$$
\begin{aligned}
\mathrm{dL}_{\mathrm{e}} / \mathrm{dX} & =2.4 \times 10^{-8} \mu \mathrm{b}^{-1}\left(\begin{array}{l}
0.5<\mathrm{X}<0.95) \\
\mathrm{x}>0.95)
\end{array}\right. \\
\text { and } \quad \mathrm{dt}_{\gamma} / \mathrm{dX} & =-2.8 \times 10^{-7} \mu \mathrm{b}^{-1} \mu \mathrm{b}^{-1} \ln \mathrm{X} / \mathrm{X}
\end{aligned}
$$

